\title{
Real-Time Surveillance of Infectious Diseases: TAIWAN's EXPERIENCE
}

\author{
Shu-Wan Jian, Chiu-Mei Chen, Cheng-Yi Lee, and Ding-Ping Liu
}

Integration of multiple surveillance systems advances early warning and supports better decision making during infectious disease events. Taiwan has a comprehensive network of laboratory, epidemiologic, and early warning surveillance systems with nationwide representation. Hospitals and clinical laboratories have deployed automatic reporting mechanisms since 2014 and have effectively improved timeliness of infectious disease and laboratory data reporting. In June 2016, the capacity of real-time surveillance in Taiwan was externally assessed and was found to have a demonstrated and sustainable capability. We describe Taiwan's disease surveillance system and use surveillance efforts for influenza and Zika virus as examples of surveillance capability. Timely and integrated influenza information showed a higher level and extended pattern of influenza activity during the 2015-16 season, which ensured prompt information dissemination and the coordination of response operations. Taiwan also has well-developed disease detection systems and was the first country to report imported cases of Zika virus from Miami Beach and Singapore. This illustrates a high level of awareness and willingness among health workers to report emerging infectious diseases, and highlights the robust and sensitive nature of Taiwan's surveillance system. These 2 examples demonstrate the flexibility of the surveillance systems in Taiwan to adapt to emerging infectious diseases and major communicable diseases. Through participation in the GHSA, Taiwan can more actively collaborate with national counterparts and use its expertise to strengthen global and regional surveillance capacity in the Asia Pacific and in Southeast Asia, in order to advance a world safe and secure from infectious disease.

Keywords: Surveillance, Infectious diseases

$T^{\prime}$ HE MOVEMENT OF INTERNATIONAL TRADE and travel worldwide has had a growing influence on global transmission of emerging infectious diseases. ${ }^{1}$ As a result, partnerships between countries with the commitment to create a world safe and secure from infectious disease threats are crucial. Taiwan, a commercial and travel hub on the Pacific Rim with a risk of imported infectious diseases such as avian influenza and Zika virus, is actively participating in this partnership by fulfilling and examining its Global Health Security Agenda (GHSA)-related capacities by

Shu-Wan Jian, MPH, is a Technical Specialist; Chiu-Mei Chen, MSPH, is a Technical Specialist; Cheng-Yi Lee, PhD, is an Associate Researcher; and Ding-Ping Liu, PhD, is Director; all at the Epidemic Intelligence Center, Centers for Disease Control, Taipei City, Taiwan.

(C) Shu-Wan Jian et al., 2017; Published by Mary Ann Liebert, Inc. This Open Access article is distributed under the terms of the Creative Commons Attribution Noncommercial License (http://creativecommons.org/licenses/by-nc/4.0/) which permits any noncommercial use, distribution, and reproduction in any medium, provided the original author(s) and the source are credited. 
using the Joint External Evaluation (JEE) tool. ${ }^{2}$ One key capacity is real-time surveillance. Surveillance is an important focus area for Taiwan, which was externally evaluated to have a demonstrated and sustainable capability to fulfill the requirements under the International Health Regulations (IHR) (2005).

In this article, we introduce the surveillance systems for infectious diseases in Taiwan. We use influenza surveillance to describe a comprehensive surveillance structure that integrates information from relevant surveillance systems, and we use Zika surveillance to address the combination of existing surveillance capacities and new strategies to respond to an ad hoc event that may constitute a public health emergency of international concern (PHEIC).

\section{Improving Infectious Disease} SuRVEILlanCE

\section{Surveillance for Notifiable Diseases}

The National Notifiable Diseases Surveillance System (NNDSS) of the Taiwan Centers for Disease Control (Taiwan CDC) has been the foundation of web-based infectious disease surveillance in Taiwan since 2001. The NNDSS is a centralized passive surveillance system that integrates reporting data and laboratory diagnosis data. Health workers are able to access the NNDSS through their medical personnel ID card, which is an official integrated circuit (IC) smart card for electronic signature issued by the Ministry of Health and Welfare (MOHW), to report suspected or confirmed cases and submit clinical specimens if necessary. In 2014, the ministry implemented the Taiwan Health Cloud program. In this program, the "communicable disease control cloud" performs automatic reporting using hospital electronic medical records (EMRs); it has improved the timeliness of reporting to the NNDSS. From 2014 to 2016, a total of 47 medical centers and metropolitan hospitals set up an automatic reporting mechanism, representing half of the records reported to the NNDSS. In addition, this system allows healthcare providers to automatically receive patient test results and reduces the need for double entry of data. For instance, after using hospital EMRs, because of the automatic integrated system to generalize patient-centered information, the average time required for a healthcare worker to complete the report process to the Taiwan CDC has been reduced from 9.2 minutes to 6.3 minutes. $^{3}$

\section{Emergency Room Surveillance}

The need to rapidly detect possible outbreaks of communicable diseases in communities, track the trends, and predict the prevalence of disease is one of the lessons learned during the SARS outbreak in 2003. ${ }^{4}$ A hospital emergency room-based real-time outbreak and disease surveillance system was established in 2004, which aims to collect individual ICD-9-CM and ICD-10 codes from patient ER visits at designated emergency response hospitals. The realtime outbreak and disease surveillance system provides realtime, comprehensive coverage, with $90 \%$ of patient record uploads to the Taiwan CDC's server occurring within 1 hour and the remaining 10\% within 1 day. It also covered more than $96 \%$ of all ER visits recorded by National Health Insurance (NHI) in Taiwan during 2012 to 2015.

In addition, since 2008, the Taiwan CDC receives aggregated numbers of outpatient, inpatient, and ER visits from NHI claims data (coverage is $>99 \%$ of the population) via machine-to-machine interface. The time between data entries from hospitals and clinics to data receipt by the Taiwan CDC is 2 days.

\section{Death Certificate Surveillance}

Since 2009, information from the National Death Certificate System has been automatically submitted to the data warehouse in the Taiwan CDC daily and is used to calculate infectious disease-related deaths. Medical institutions report this information to the system within 7 days of issuance of a death certificate. In 2014, the death records from this system covered $87.2 \%$ of all the deaths published by the Department of Statistics.

\section{Laboratory Surveillance}

The national laboratory surveillance network monitors viral activity in the community and is the foundation of influenza and enterovirus surveillance. From 2014 to 2016, the laboratory automated reporting system (LARS) was developed to improve efficiency and representativeness of community-based laboratory reporting for 20 microorganisms of public health concern. The reporting system uses a database and universal standard for identifying medical laboratory observations called Logical Observation Identifiers Names and Codes (LOINC) to automatically and daily submit information on positive results from the laboratory information management system (LIMS) used in clinical laboratories. By adopting data standards and formats for automated laboratory reporting, 51 medical centers and metropolitan hospitals are able to submit laboratory information to the laboratory automated reporting system. The information from hospitals, delivered via a cloud data exchange platform, are submitted to the data warehouse of the Taiwan CDC and converted to an open data format for dissemination through the national open data platform (Figure 1).

\section{Event-Based Surveillance}

The rapid detection, reporting, confirmation, and assessment of events that are a potential risk to public health are 


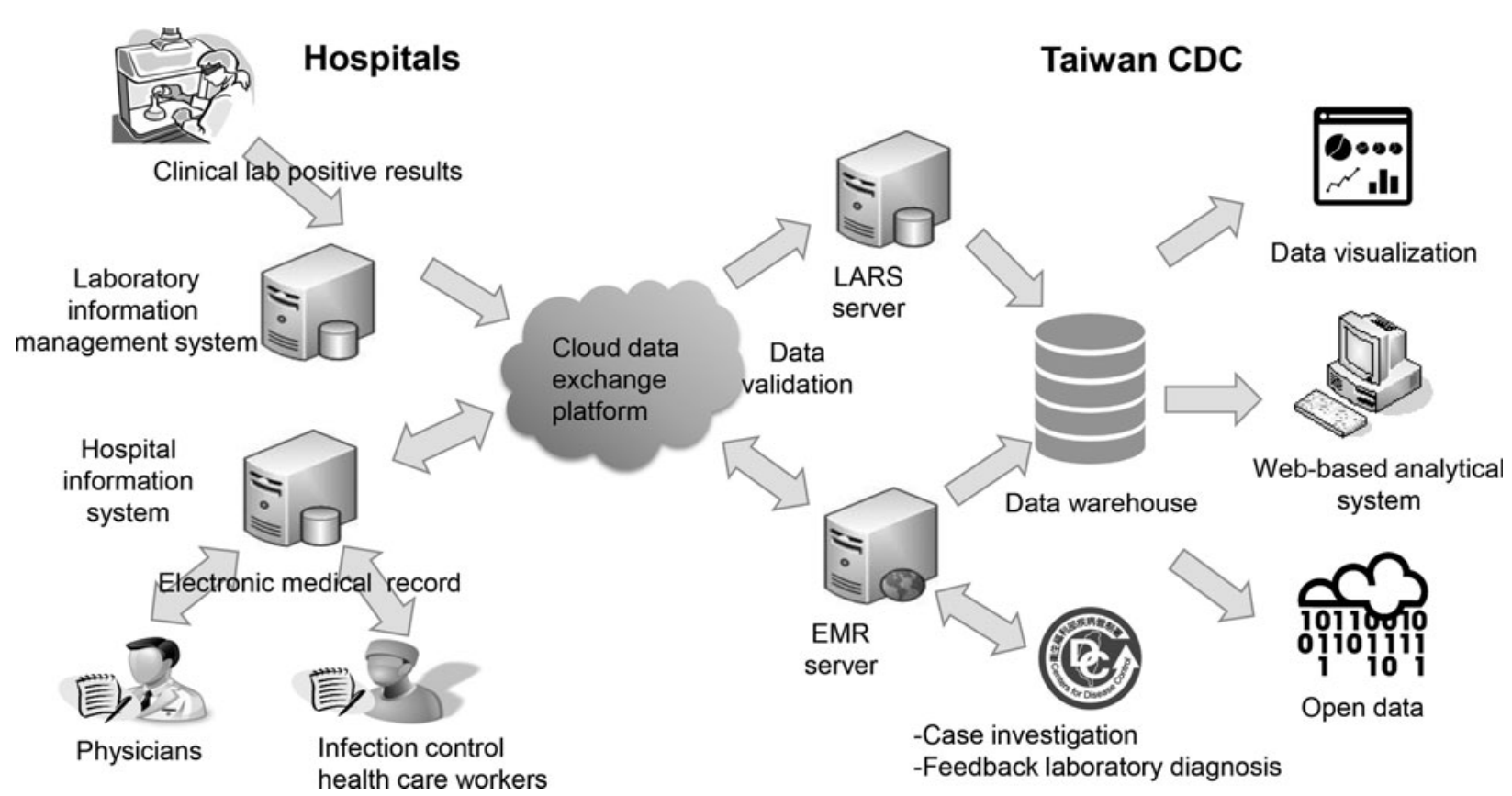

Figure 1. Schematic diagram of Taiwan's hospital information system (HIS) and laboratory information management system (LIMS), used to report infectious diseases and release data

established practice at the Taiwan CDC. Sources of information include newspapers, web browser queries, social media, TV channels, e-newspapers (which deliver domestic and international news to internal subscribers twice daily), official and nonofficial websites of public health bodies, diplomatic telegrams, fax and toll-free communicable disease reporting, and a consultation hotline. In addition, scientific articles and confidential information from the WHO event information services and international public health bodies are also included to ensure information verification. This rapid response capacity is used to immediately communicate with the public about rumors and verified events on the official website of the Taiwan CDC. In addition, an epidemic intelligence threat report is disseminated daily for internal use. As of November 1, 2016, the event-based surveillance at the Taiwan CDC has tracked more than 1,900 events in more than 170 countries, and it monitors approximately 3 to 10 public health threats each day.

\section{Information Availability to the Public}

The Taiwan CDC open data portal provides more than 200 datasets, including all notifiable disease cases and emergency department visits for selected syndromes, and information on quarantine practices, vaccines, nosocomial infections, and the like. Most of the information-for example, datasets related to number of cases of all notifiable diseases and emergency department visits-are updated automatically and daily. New datasets are publicly posted after discussion and meet the principles of open data. The open data portal can be accessed by the public, and the datasets have been downloaded more than 220,000 times in 2016. ${ }^{5,6}$ The Taiwan national infectious disease statistics system is a public website that provides nationwide data on confirmed cases and syndromic data by disease category, jurisdiction, time period, and case origin, and generates charts automatically after queries. The statistics are updated automatically on a daily basis, and raw data can be downloaded in CSV or Excel files for further applications. ${ }^{7}$

\section{EXAmple 1: SurveillanCe fOR INFluenZA}

Influenza surveillance in Taiwan is a collaborative effort among the department of statistics at the ministry of health, the National Health Insurance administration, local departments of health, the National Influenza Center, regional commissioned laboratories for influenza virus, and healthcare facilities. Information on influenza activity includes the percentage of positive specimens for influenza; viral type and antigenicity; antiviral resistance; outpatient, inpatient, and hospital visits for influenzalike illness (ILI) surveillance; severe complicated influenza notification; pneumonia; and influenza mortality surveillance. ${ }^{8-10}$ This information is essential for risk assessment and estimations of morbidity, mortality, and the potential impact of influenza in the community and on healthcare services. The Taiwan CDC monitors influenza and other respiratory disease activities year-round and produces Taiwan Influenza Express, a weekly influenza surveillance online report, during the influenza season (October [week 40] to May [week 20]). ${ }^{11}$ The integration of surveillance systems to 
detect an influenza outbreak during 2015-16 influenza season is shown as an example below.

\section{Influenza Laboratory Surveillance}

The laboratory surveillance network is made up of 8 regional commissioned laboratories. The specimens are cultured to identify the subtype and antigenicity of influenza virus, with up to a 2-week lag between the date of specimen receipt and the date of submitting diagnostic results to the Taiwan CDC. In 2014, the development of the "communicable disease control cloud" established the laboratory automated reporting system, in which the 51 clinical laboratories automatically submit daily positive results tested by rapid influenza diagnostic tests, polymerase chain reaction (PCR), and enzyme immunoassay (EIA) to detect viral antigens. ${ }^{12}$ Figure 2 shows the number of influenza-positive specimens reported by the laboratory automated reporting system (A) and the commissioned laboratories (B) between week 40, 2015, and week 40, 2016. A total of 1,578 influenza-positive specimens were reported by the 8 commissioned laboratories between week 40, 2015, and week 40, 2016 (Figure 2A). Compared to the commissioned laboratory surveillance network, the total number of influenza-positive specimens from the laboratory automated reporting system of the same period was 48,206 . The laboratory reporting system could track a greater number of positive specimens, and the trend is relatively smoother (Figure 2B).

In addition, the laboratory automated reporting system detected cases of type B influenza exceeding half of all the positive specimens in week 10,2016 , which was 2 weeks earlier than the trend detected by the regional commissioned laboratories. The laboratory automated reporting system helps to provide real-time reporting, enabling rapid implementation of control measures for influenza outbreaks, while the commissioned laboratories provide the information on influenza viral subtype and antigenic characteristics. The integration of the 2 surveillance systems gives a comprehensive picture of the influenza viral characteristics.
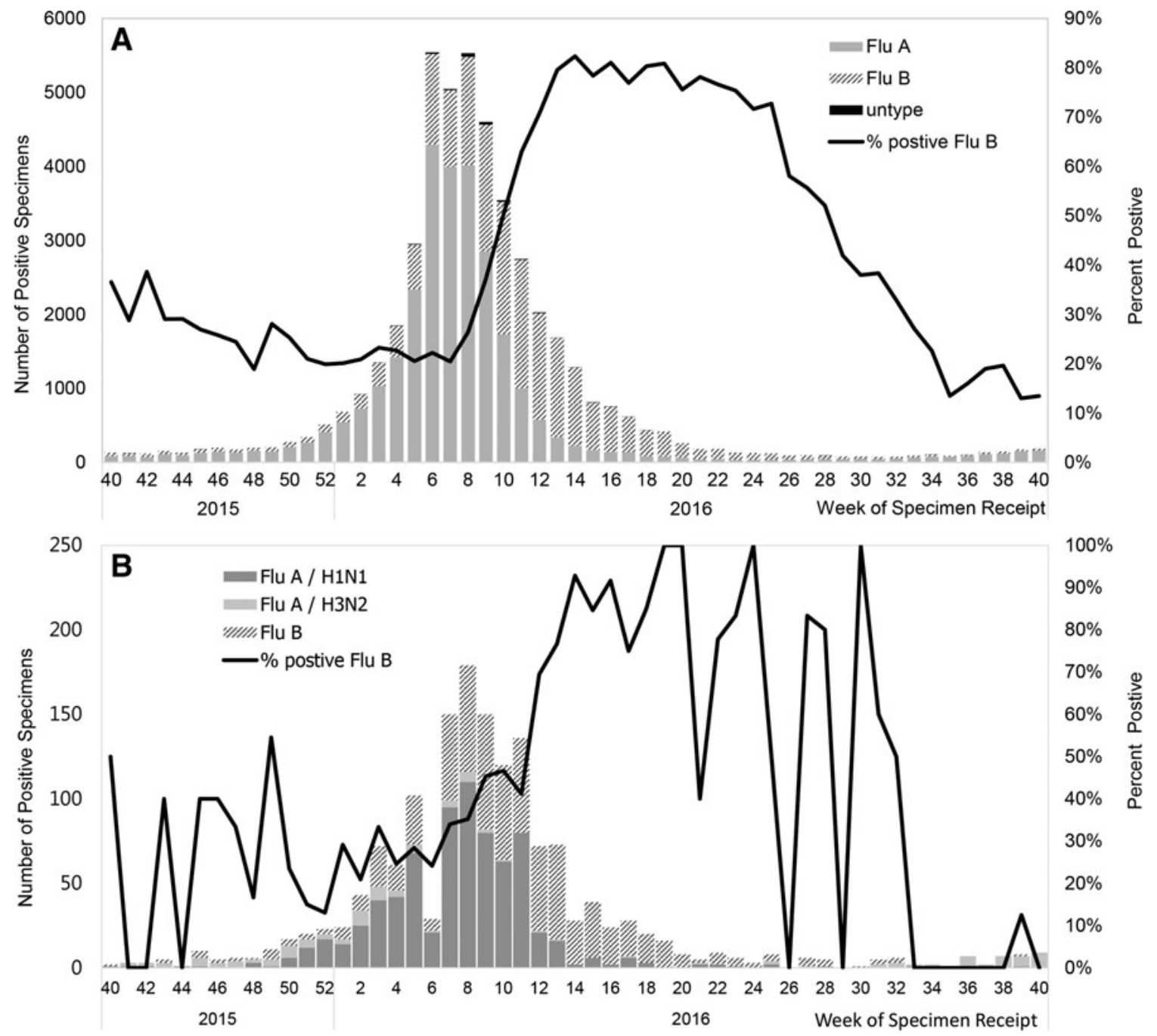

Figure 2. The influenza laboratory surveillance in Taiwan: laboratory automated reporting system (LARS) (A) and the laboratory surveillance network (B), week 40, 2015 to week 40, 2016. 


\section{Influenzalike Illness Syndromic Surveillance}

ILI patients seeking care at ERs are detected through a set of ICD-9-CM/ICD-10 codes for respiratory syndromes monitored through the real-time outbreak and disease surveillance system. The National Health Insurance administration collects ICD-9-CM (code: 480-487) and ICD-10 (code: J12J17, B25, A37, A22, B44) diagnostic codes from ILI outpatient records. The percent of ER and outpatient visits due to ILIs tended to increase each year at approximately weeks 48 to 51 and decline at weeks 9 to 10 in the following year during the 2013 to 2016 influenza seasons (Figure 3). Compared to the previous $2 \mathrm{flu}$ seasons, the influenza outbreak occurring from mid-2015 to mid-2016 was the most serious outbreak, with an extended pattern. Both real-time outbreak and disease surveillance system and National Health Insurance data detected increased $\mathrm{flu}$ activities starting in week 46, peaked during week 9, 2016, and then declined.

\section{Hospitalized and Severe Influenza Case Surveillance}

The National Health Insurance system and NNDSS tracking of hospitalized influenza cases (ICD-9-CM code: 487 and ICD-10 code: J09-J11) and severe complicated influenza cases, respectively, detected trends of increasing cases around weeks 50 to 52 and declines in weeks 9 to 10 in the following year during the 2013 to 2016 influenza seasons (Figure 4). An unprecedented number of severe complicated influenza cases $(n=2,018)$ were reported in the influenza season from July 1, 2015, to June 30, 2016; $95 \%$ of those had not received the $2015-16 \mathrm{flu}$ vaccine, $75.6 \%$ were infected with $\mathrm{H} 1 \mathrm{~N} 1$, and $12.9 \%$ were infected with type $B$ influenza. In addition, when compared to the previous 2 influenza seasons, the age group with the highest incidence was aged $\geq 65$ years, and the highest number of cases was among those aged 50 to 64 (Figure 5). Another observation was that the rate of change in incidence of severe complicated influenza per 100,000 population was most significant among people aged 50 to 64 years, compared to the other age groups during the 2015-16 influenza season.

\section{Pneumonia and Influenza Mortality Surveillance}

Trends in pneumonia and influenza deaths are identified by searching the National Death Certificate System data for the keywords pneumonia, common cold, or influenza. To ensure data stability, 4-week moving averages are used as a standard for calculating weekly surveillance results. There were approximately 300 to 400 pneumonia and influenza deaths per week during the nonepidemic period, with

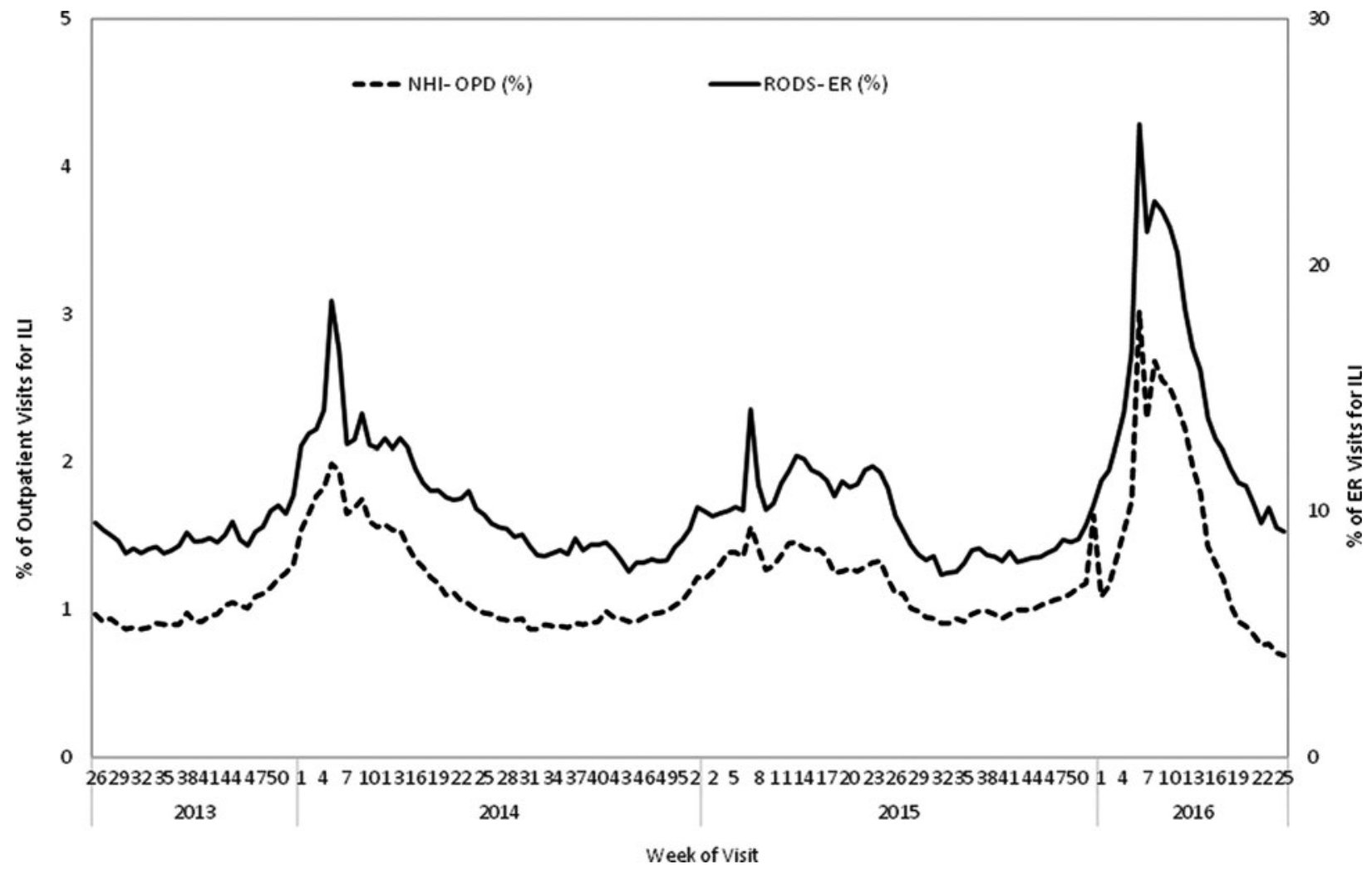

Figure 3. Percentage of visits for influenzalike illness (ILI) in emergency rooms (monitored through RODS) and outpatient departments (OPD, monitored through NHI), week 26, 2013, to week 25 in Taiwan, 2016. 
JIAN ET AL

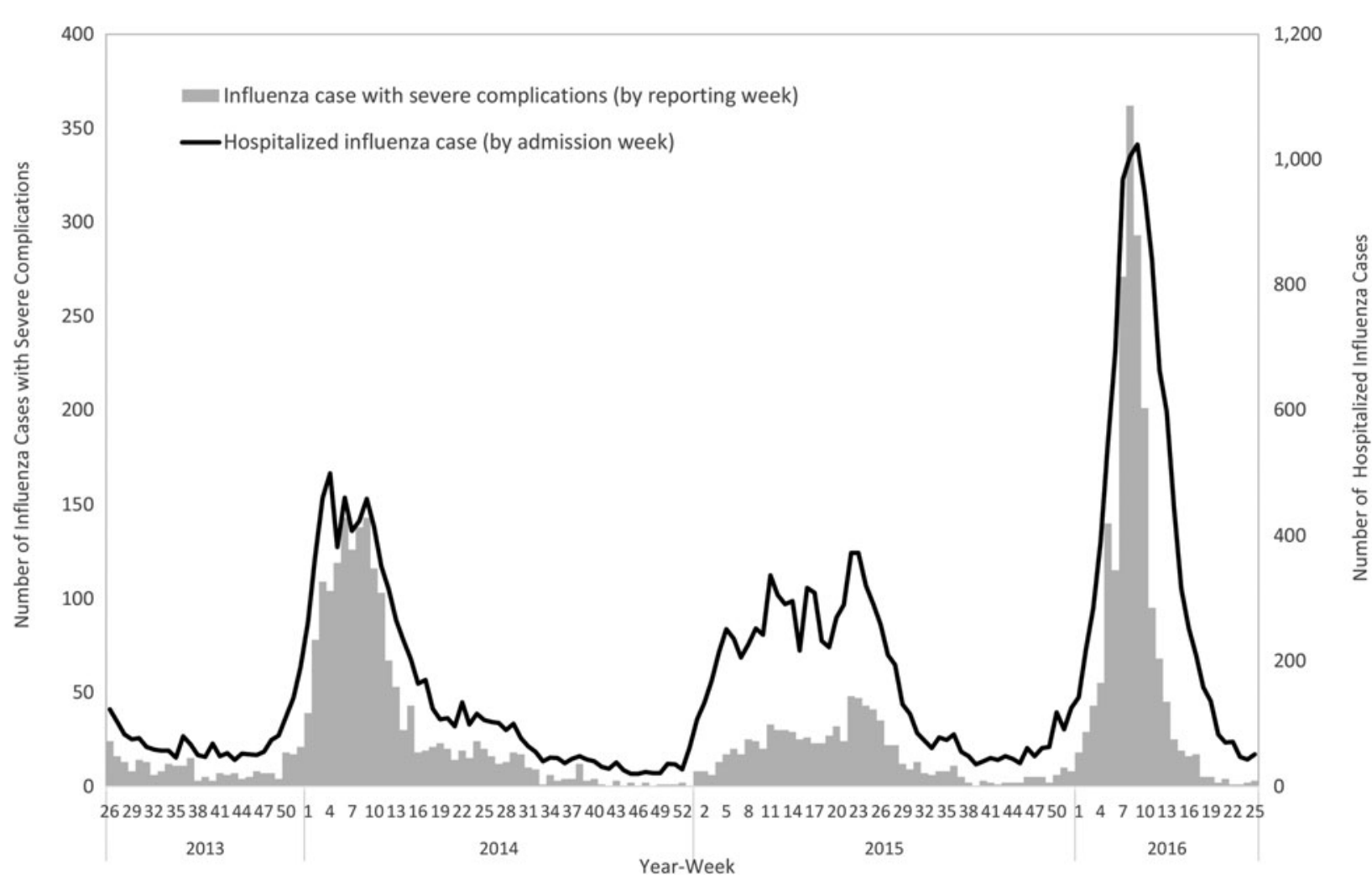

Figure 4. Number of severe complicated influenza cases and influenza-associated hospitalizations from week 26, 2013, to week 25, 2016, in Taiwan

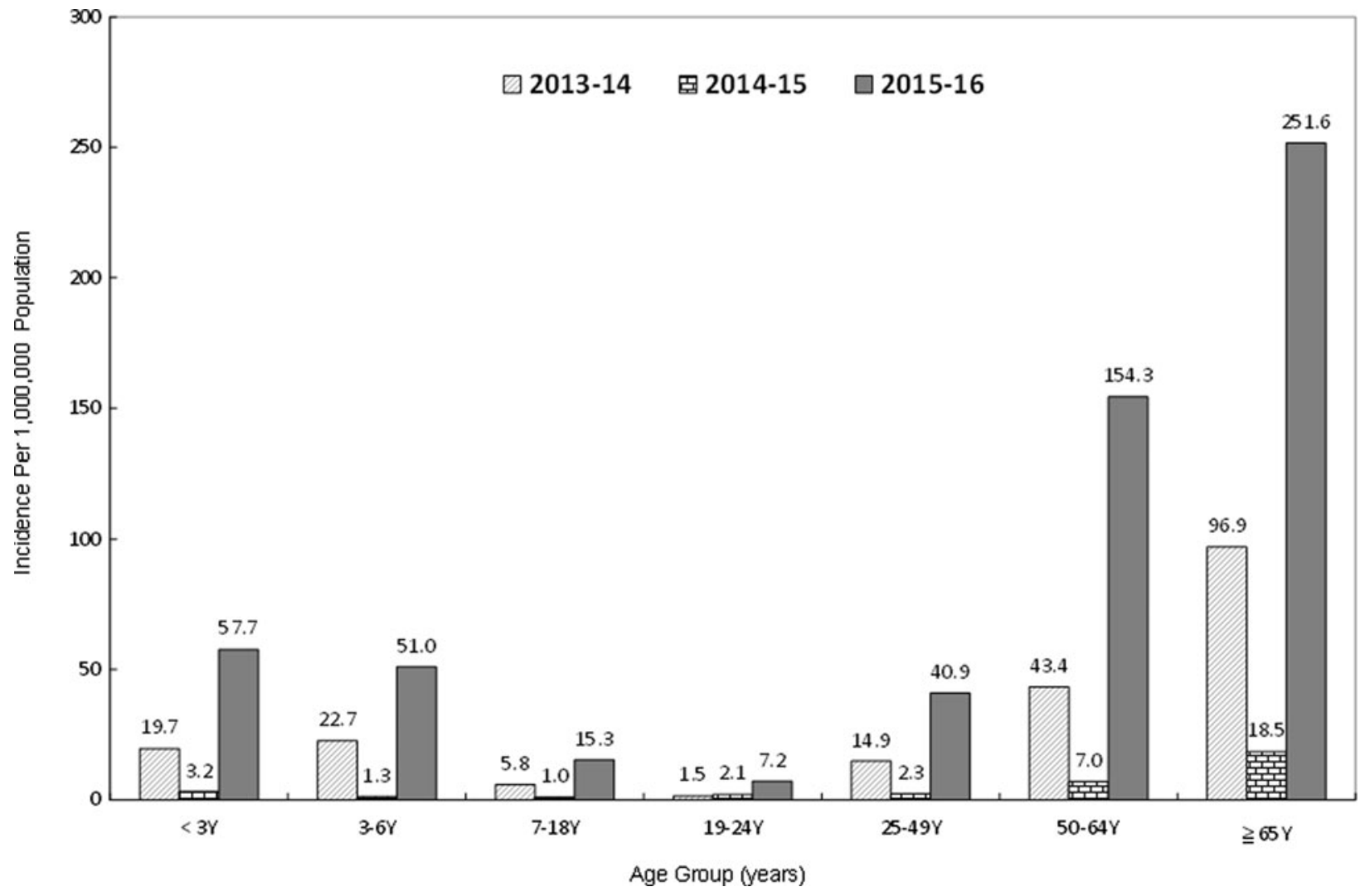

Figure 5. The incidence of severe complicated influenza by age group during 2013 to 2016 influenza seasons 
pneumonia and influenza deaths increasing greatly during the epidemic season. Reporting of these data is delayed by only 1 week or less from the time a death certificate is issued, with $>85 \%$ coverage of all death records. As a result, this system is able to help track epidemics and measure the severity of an influenza season, which helps to support strategies and communication with the public. In the 201516 influenza season, the number of pneumonia and influenza deaths increased from week 50 and peaked during week 9 in the following year. It was much higher than the preceding 2 seasons (2013-14 and 2014-15) (Figure 6).

\section{Example 2: Surveillance for Zika Virus}

Taiwan established border health practices beginning in 2003. These practices involve entry screening for fever, traveler self-reporting of symptoms, and messages of personal health education on infectious diseases. Passengers with fever who are from regions with endemic or epidemic dengue may also be tested for dengue and flavivirus infection.

On January 10, 2016, the first imported case of Zika virus infection in Taiwan was detected through screening for fever at Taoyuan International Airport. The event, and the subsequent declaration of Zika as a public health emergency of international concern, triggered the following enhanced measures to detect Zika virus: ${ }^{13}$

1. Zika virus infection was listed as a category 5 notifiable disease based on the Communicable Disease Control Act. As a result, any suspected infected persons identified by physicians had to be reported within 24 hours and further case investigation would be completed within the next 24 hours.

2. The Central Epidemic Command Center was activated at level 3, indicating that Zika is a public health emergency of international concern, and an imported case was detected in Taiwan. The director general of the Taiwan CDC is the commander during level 3 activation and coordinates related government agencies, such as the Environmental Protection Agency and the Ministry of Education.

3. A laboratory diagnosis network was set up for Zika virus. This network includes 9 clinical laboratories and the national laboratory at the Taiwan CDC. The designated clinical laboratories are able to detect Zika virus by realtime PCR to confirm viremic Zika patients using blood and urine specimens. As of November 5, the average interval from date of reporting to date of laboratory diagnosis is 2 to 3 days, which is rapid enough to enable effective environmental and vector control measures.

4. Free testing was established for pregnant women with possible exposure to Zika virus, regardless of the development

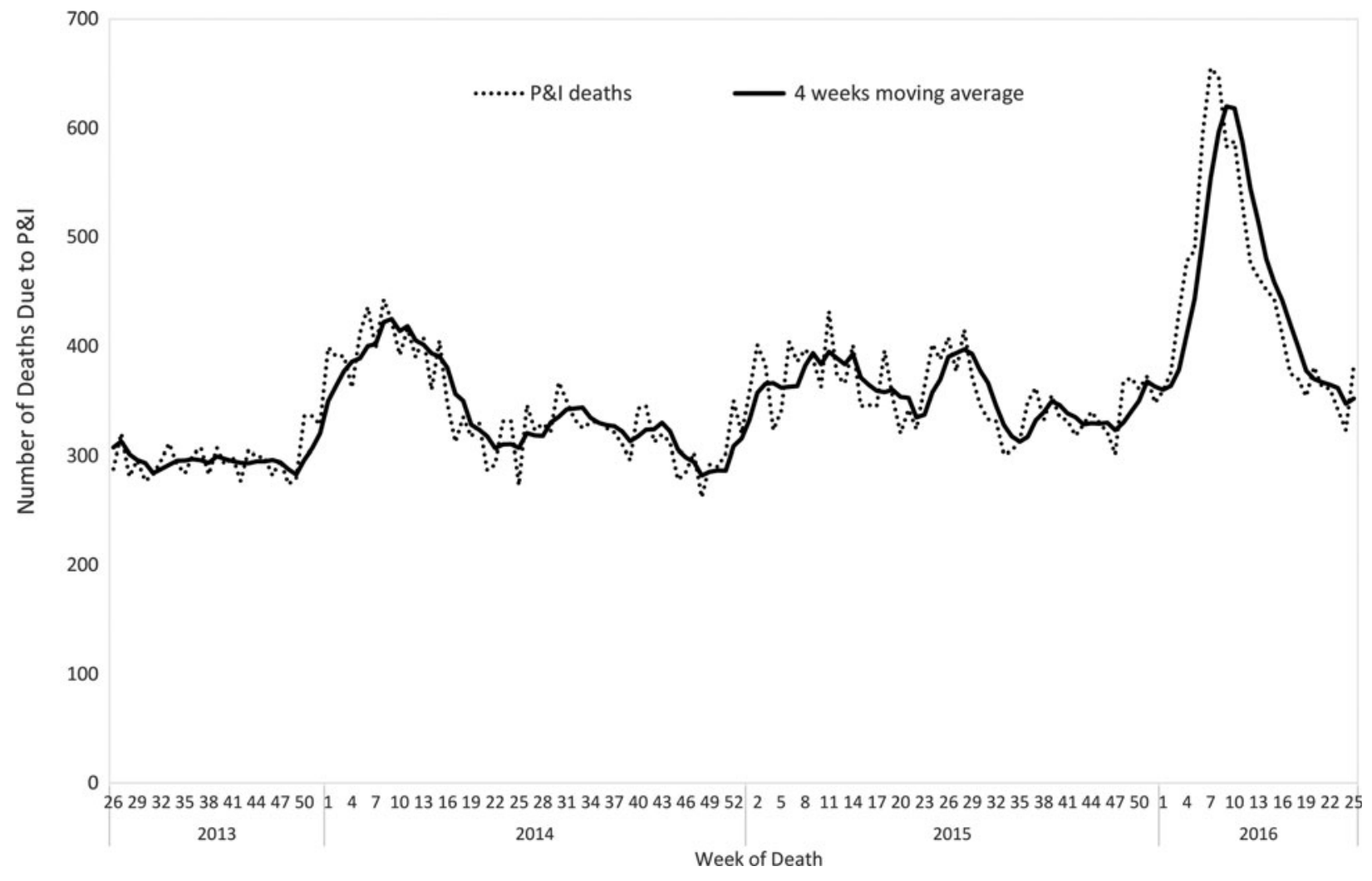

Figure 6. Pneumonia and influenza mortality from week 27, 2013, to week 26, 2016, in Taiwan 
JIAN ET AL

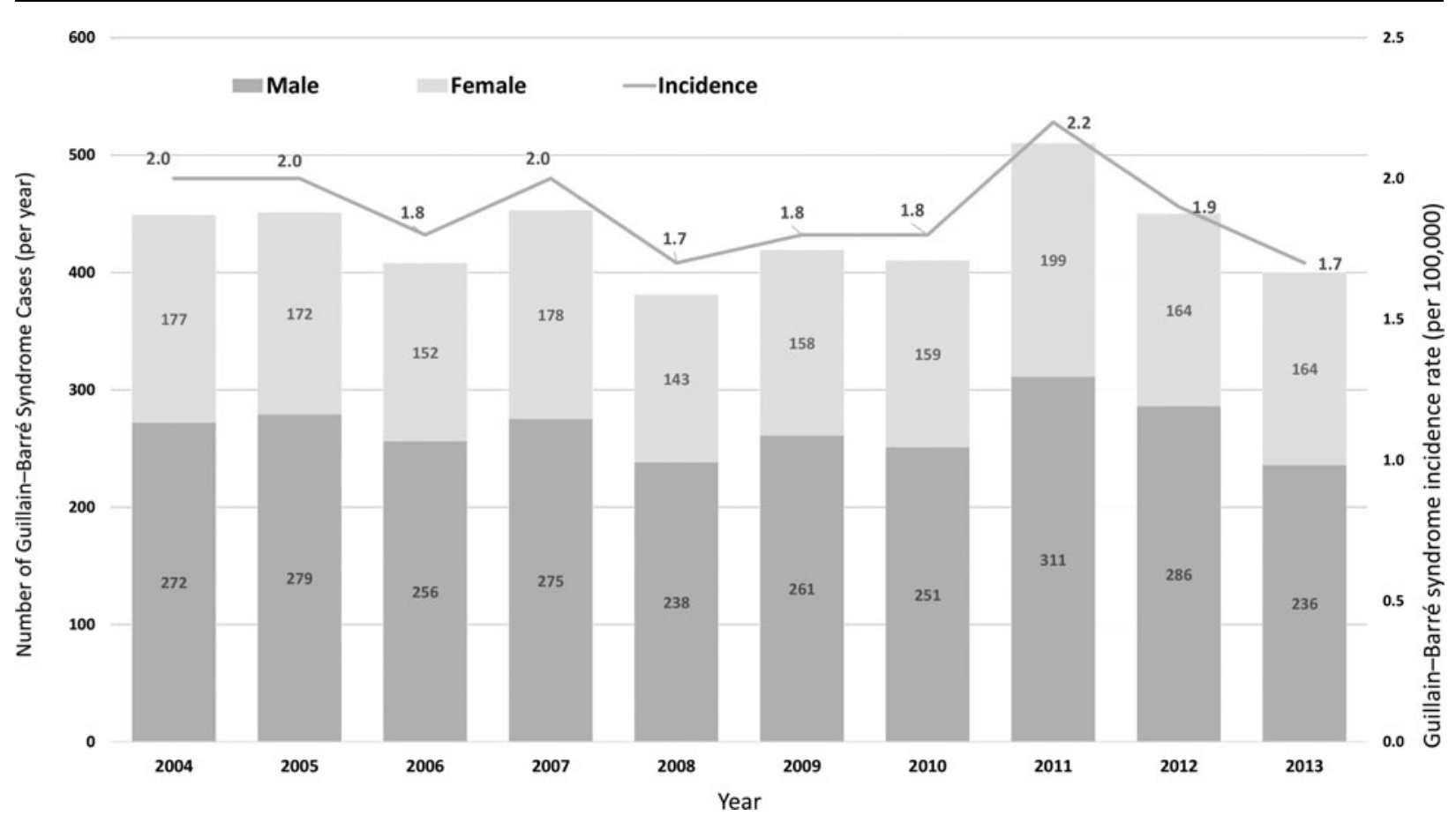

Figure 7. The incidence and number of Guillain-Barré Syndrome (GBS) cases in Taiwan, 2004-2013

of symptoms. Tests can be requested via a self-report using the Taiwan CDC's toll-free hotline or through a physician.

As of November 5, 2016, 13 imported cases (and no indigenous cases) have been detected in Taiwan from countries with high Zika risk, including Indonesia, Malaysia, Singapore, Thailand, Vietnam, Saint Lucia, Saint Vincent and the Grenadines, and the United States. Taiwan was the first country to report imported cases from Miami Beach and Singapore, highlighting the robust and sensitive nature of Taiwan's surveillance system. ${ }^{14,15}$ Taiwan's vector surveillance programs have identified Aedes albopictus in all regions in Taiwan and Aedes aegypti in southern Taiwan, highlighting the risk of domestic transmission of the Zika virus. ${ }^{16}$ However, detection of indigenous Zika cases relies on healthcare workers with a high awareness identifying a patient presenting with Zika symptoms but no travel history during the patient's incubation period. ${ }^{17}$

The WHO scientific committee concurred with the international scientific consensus that Zika virus is a cause of microcephaly and Guillain-Barré syndrome (GBS). ${ }^{18}$ One of the surveillance and response indicators developed by WHO for global response to the Zika virus outbreak is to establish a baseline for GBS at a country level, which could become a surrogate for the GBS surveillance and capacity to detect an increase in the incidence of GBS. ${ }^{19}$

In addition to conducting surveillance for symptomatic Zika cases, the Taiwan CDC also monitored GBS to determine the effect of Zika virus infection. As a baseline value in Taiwan, we reviewed the hospitalization records of the National Health Insurance database between 2004 and 2013 to estimate the rate of GBS morbidity. Surveillance of microcephaly will be included by connecting the database of National Health Insurance in 2017. We defined a GBS patient as a hospitalized patient with ICD-9-CM code 357.0. An estimated 381 to 510 persons, or 1 to 2 persons per 100,000, developed GBS from 2004 to 2013, which is similar to the morbidities in retrospective database studies in the US, Canada, and Europe (Figure 7). ${ }^{20-25}$ The overall male-to-female ratio is 1.60 , and the major GBS cases were in people ages 19 to 50 and 51 to 64 (data not shown). This gives baseline information on the GBS incidence, which will provide important information for Zika surveillance in the future.

\section{Conclusion}

Using technology to comprehensively integrate indicatorbased, event-based, and syndromic surveillance systems has strengthened detection of infectious diseases in Taiwan at all levels. This provides real-time, accurate clinical, epidemiologic, and laboratory information but maintains the flexibility to adapt surveillance systems to both emerging infectious diseases and major communicable diseases. Taiwan has the capability to report a public health emergency of international concern event such as a Zika case to WHO within 24 hours, as required under IHR (2005), and can implement rapid response control measures.

Taiwan is planning its roadmap to engage and improve all action packages of the JEE tool. It will also work to strengthen interagency accountability and collaboration on achieving a One Health approach and GHSA targets. This 
includes the mechanism of sharing data by using interoperable electronic reporting in public health surveillance systems between different sectors and developing an early warning system for zoonotic diseases.

As a country with experience in responding to and preparing for SARS and avian influenza, through participation in the GHSA, Taiwan can more actively collaborate with its national counterparts in public health and use its expertise to strengthen both the global and regional capacity in the Asia Pacific and in Southeast Asia, to respond to threats of infectious diseases, prevent disease transmission, and ensure global health security.

\section{ACKNOWLEDGMENT}

We acknowledge the assistance of the officers at the epidemic intelligence center, Taiwan CDC, who contributed their time to provide insights during the writing of this article.

\section{REFERENCES}

1. Relman DA, Choffnes ER, Mack A, rapporteurs; Institute of Medicine; Board on Global Health; Forum on Microbial Threats. Infectious Disease Movement in a Borderless World: Workshop Summary. Washington, DC: National Academies Press; 2010.

2. Centers for Disease Control, R.O.C. (Taiwan). As imported dengue cases continue to be reported, Taiwan CDC urges travelers to seek immediate medical attention when feeling unwell upon arriving in Taiwan to prevent further transmission [press release]. August 10, 2016. http://www. cdc.gov.tw/english/info.aspx?treeid=bc2d4e89b154059b\&

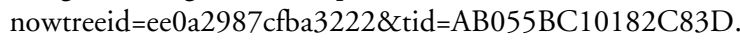
Accessed November 23, 2016.

3. Hu YP, Li CL, Liu YL, Chang CM, Chuang JH. Reporting notifiable infectious diseases by using hospital electronic medical record systems: An effectiveness assessment. Journal of Taiwan Association for Medical Informatics 2016;25(1):23-32.

4. Wu TS, Shih FY, Yen MY, et al. Establishing a nationwide emergency department-based syndromic surveillance system for better public health responses in Taiwan. BMC Public Health 2008;8:18.

5. Taiwan CDC open data portal and platform statistics. https://data.cdc.gov.tw/en/. Accessed January 16, 2017.

6. Government open data portal. http://data.gov.tw/. Accessed January 16, 2017.

7. Taiwan National Infectious Disease Statistics System. http:// nidss.cdc.gov.tw/en/. Accessed January 16, 2017.

8. Lai SK, Chang HL, Wu HS, Chuang JH. Multiple disease surveillance systems against pandemic (H1N1) influenza in Taiwan. Taiwan Epidemiol Bull 2010;26(14):251-261.

9. Chuang JH, Huang AS, Huang WT, et al. Nationwide surveillance of influenza during the pandemic (2009-10) and post-pandemic (2010-11) periods in Taiwan. PLoS One 2012; 7(4):e36120.
10. Wu WJ, Chang CM, Jiang CH, Yang SL, Chuang JH. Realtime surveillance of pneumonia and influenza mortalities via the National Death Certificate Systems. Taiwan Epidemiol Bull 2009;25(10):727-738.

11. Centers for Disease Control, R.O.C. (Taiwan). Influenza express. http://www.cdc.gov.tw/english/submenu.aspx?treeid= 00ed75d6c887bb27\&nowtreeid=8f1e239d0fd8877a. Accessed November 23, 2016.

12. Centers for Disease Control, R.O.C. (Taiwan). Applications of using cloud computing for communicable disease surveillance. 2015. http://www.cdc.gov.tw/professional/ programresultinfo .aspx?treeid $=9068 \mathrm{acd} 483 \mathrm{c} 71 \mathrm{fc} 1$ \&nowtreeid= 3B791EACC1B5C579\&tid=AB4E7C296841EC2D. Accessed November 23, 2016.

13. World Health Organization. WHO statement on the first meeting of the International Health Regulations (2005) (IHR 2005) Emergency Committee on Zika virus and observed increase in neurological disorders and neonatal malformations. February 1, 2016. http://www.who.int/mediacentre/news/ statements/2016/1st-emergency-committee-zika/en/. Accessed November 23, 2016.

14. Centers for Disease Control, R.O.C. (Taiwan). Taiwan confirms sixth imported Zika case in Taiwanese traveler who visited Miami, Florida [press release]. August 17, 2016. http://www.cdc.gov.tw/english/info.aspx?treeid=bc2d4e89b 154059b\&nowtreeid=ee0a2987cfba3222\&tid=E285278D5 A2EE5A6. Accessed November 23, 2016.

15. Centers for Disease Control, R.O.C. (Taiwan). Taiwan confirms seventh imported Zika case in Singaporean, which is the first imported case from Singapore [press release]. September 12, 2016. http://www.cdc.gov.tw/english/info. aspx?treeid=bc2d4e89b154059b\&nowtreeid=ee0a2987cfba 3222\&tid=EB586FD553EC4297. Accessed November 23, 2016.

16. Kraemer MUG, Sinka ME, Duda KA, et al. The global compendium of Aedes aegypti and Ae. albopictus occurrence. Sci Data 2015;2:150035.

17. Jian SW, Lin CL, Liu DP. Risk assessment on Zika virus infection in Taiwan. Taiwan Epidemiol Bull 2016;32(11):225235. http://www.cdc.gov.tw/english/info.aspx?treeid=38477 $19104 \mathrm{be} 0678 \&$ nowtreeid $=05056 \mathrm{~b} 75 \mathrm{c} 73 \mathrm{beba} 6 \&$ tid $=\mathrm{BD} 377$ 60C1102ED4F. Accessed November 23, 2016.

18. World Health Organization. Zika situation report. January 16, 2016. http://www.who.int/emergencies/zika-virus/situationreport/16-june-2016/en/. Accessed November 23, 2016.

19. World Health Organization. Zika virus outbreak global response. July 15, 2016. http://www.who.int/emergencies/zikavirus/response/en/. Accessed November 23, 2016.

20. Alshekhlee A, Hussain Z, Sultan B, Katirji B. Guillain-Barré syndrome: incidence and mortality rates in US hospitals. Neurology 2008;70(18):1608-1613.

21. US Centers for Disease Control and Prevention. Zika and Guillain-Barré syndrome. CDC website. August 9, 2016. http://www.cdc.gov/zika/healtheffects/gbs-qa.html. Accessed November 23, 2016.

22. McLean M, Duclos P, Jacob P, Humphreys P. Incidence of Guillain-Barré syndrome in Ontario and Quebec, 19831989, using hospital service databases. Epidemiology 1994; 5(4):443-448.

23. Hauck LJ, White C, Feasby TE, Zochodne DW, Svenson LW, Hill MD. Incidence of Guillain-Barré syndrome in 
Alberta, Canada: an administrative data study. I Neurol Neurosurg Psychiatry 2008;79(3):318-320.

24. Benedetti MD, Pugliatti M, D’Alessandro R, et al. A multicentric prospective incidence study of Guillain-Barré syndrome in Italy. The ITANG Study. Neuroepidemiology 2015; 45(2):90-99.

25. McGrogan A, Madle GC, Seaman HE, de Vries CS. The epidemiology of Guillain-Barré syndrome worldwide. A systematic literature review. Neuroepidemiology 2009;32(2): 150-163.
Manuscript received December 18, 2016;

revision returned January 26, 2017;

accepted for publication February 8, 2017.

Address correspondence to: Ding-Ping Liu, PhD

Director, Epidemic Intelligence Center

Centers for Disease Control

Taipei City, Taiwan

E-mail: dpliu@cdc.gov.tw 